\title{
A Novel Topological Multicast Routing Algorithm (ToMuRo)
}

\author{
R. Aquino-Santos*1, L. A. Villaseñor-González ${ }^{2}$, V. Rangel-Licea ${ }^{3}$, A. González-Potes ${ }^{4}$, M.A. García-Ruiz ${ }^{1}$, \\ A. Edwards-Block ${ }^{1}$ \\ ${ }^{1}$ Facultad de Telemática, Universidad de Colima, C. P. 28045, Colima, Colima, México \\ *aquinor@ucol.mx \\ ${ }^{2}$ Departamento de Electrónica y Telecomunicaciones, CICESE, \\ Carr. Tijuana-Ensenada, Km. 113, Ensenada, B. C. N., México. \\ ${ }^{3}$ Departamento de Telecomunicaciones, Universidad Nacional Autónoma de México, México, D. F. \\ ${ }^{4}$ Facultad de Ingeniería Mecánica y Eléctrica, Universidad de Colima. Coquimatlán, Colima, México
}

\begin{abstract}
This paper presents a performance analysis of an enhanced version of the Topological Multicast Routing Algorithm (ToMuRo) on mobile wireless ad-hoc networks, which includes undecided border nodes. Employing undecided border nodes to forward multicast packets represents a significant enhancement because it optimizes the path discovery process by selecting undecided nodes that can function as multicast relay nodes. Flooding Mechanism and On-Demand Multicast Routing Protocol (ODMRP) are simulated and compared with both the basic and enhanced versions of the Topological Multicast Routing algorithm. The scenario evaluated considers one multicast transmitter and one, two, and three multicast receivers with various mobility patterns and transmission ranges. The behavior of 250 nodes is evaluated in terms of End to End Delay (EED), jitter, packet delivery ratio, and overhead. Results reveal that the enhanced version of ToMuRo performs better in terms of packet delivery ratio and jitter, while ODMRP performs better with respect to EED and Overhead.
\end{abstract}

Keywords: Multicast routing algorithms, multi-hop wireless networks, ad-hoc networks, topological routing algorithm.

\section{RESUMEN}

Este trabajo presenta un análisis del funcionamiento de la versión mejorada del algoritmo de enrutamiento de difusión múltiple topológico (ToMuRo) en redes inalámbricas ad hoc móviles que incluye nodos de frontera no-decididos. La utilización de los nodos de frontera no-decididos evita que se reinicie el proceso de descubrimiento de ruta al seleccionar nodos no-decididos que puedan funcionar como nodos relevadores. El mecanismo de inundación (flooding) y el protocolo de enrutamiento de difusión múltiple bajo demanda (ODMRP) son simulados y comparados con las versiones básica y mejorada del algoritmo de enrutamiento de difusión múltiple topológico. El escenario evaluado considera un transmisor de difusión múltiple y uno, dos y tres receptores de difusión múltiple bajo varios patrones de movilidad y rangos de transmisión. El comportamiento de 250 nodos es evaluado en términos del retardo punto a punto (EED), distorsión de fase (jitter), porcentaje de entrega de paquetes, y sobre-procesamiento. Resultados revelan que la versión mejorada de ToMuRo funciona mejor en términos del porcentaje de entrega de paquetes, y distorsión de fase. ODMRP funciona bien con respecto al retardo punto a punto y sobre procesamiento.

\section{Introduction}

Multicast routing algorithms represent an important area of study in the field of wireless adhoc networks and present an important challenge because of the need to provide communication and coordination among nodes in highly mobile or dynamic settings. Furthermore, using multicast routing strategies offers the important advantage of transmitting a single packet to multiple receptor nodes simultaneously, which is extremely important in ad-hoc environments, where bandwidth resources need to be optimally exploited. Applications requiring multicasting (Mobile Learning, Voice/Video over IP, Multimedia broadcasting) are becoming increasingly common. 
One major impediment, however, is that nodes in multicast networks move omni-directionally, causing frequent and unpredictable topological changes, requiring the development of more efficient predictive algorithms. In conventional adhoc environments, network hosts work in pairs to carry out specific tasks. Multicast network algorithms, however, must simultaneously transmit information packets to several hosts, and these hosts must seamlessly discern if their role is to receive or forward the packets. Although multicast network algorithms are highly desirable for many applications, they are significantly less efficient because of their forwarding mechanism and network resource use. Packet delivery ratio, jitter and end-to-end delay are the three principal parameters taken into account when considering QoS applications and network resource management.

Current literature proposes several multicast routing algorithms for ad-hoc wireless networks, and these algorithms are categorized as either mesh based or tree based. Tree- based multicast routing algorithms offer a single path between any source/receiver pair, which uses fewer resources than mesh-based multicast routing algorithms. On the other hand, mesh-based multicast routing algorithms may maintain more than one path between a source/receiver pair, thus providing alternative routes that support packet transference redundancy.

Multicast Protocols developed for static networks (e.g. Distance Vector Multicast Routing Protocol (DVMRP) [1], Multicast Open Shorted Path First (MOSPF) [2], Core Based Trees (CBT) [3], and Protocol Independent Multicast (PIM) [4], do not perform very well in ad-hoc network environments, due to their continuous dynamic changes. One major disadvantage of the abovementioned multicast protocols is their inherently volatile tree structure, which obliges networks to continuously update their link status in response to connectivity changes. Another disadvantage is that typical multicast trees usually require a global routing substructure such as a link state or distance vector, which can result in significant packet loss. Additionally, the frequent exchange of routing vectors or link state tables, caused by continuous topology changes, can also yield excessive channel and processing overhead, resulting in significant network congestion. Finally, restraints related to limited bandwidth, power consumption, and host mobility makes multicast protocol design particularly challenging.

In response to these difficulties, several multicast routing protocols have been proposed for wireless ad hoc networks, including: Ad Hoc Multicast Routing Protocol (AMRoute) [5], On-Demand Multicast Routing Protocol (ODMRP) [6], Ad hoc Multicast Routing protocol utilizing Increasing idnumberS (AMRIS) [7], The Core-Assisted Mesh Protocol (CAMP) [8], Multicast Ad Hoc On-Demand Distance Vector (MAODV) [9], and Adaptive Demand-Driven Multicast Routing protocol (ADMR) [10]. This work presents the performance of topological multicast routing algorithms on mobile wireless ad-hoc networks using Flooding and ODMRP, which are simulated and compared with the basic and enhanced Topological Multicast Routing Protocol (ToMuRo).

The remainder of this paper is organized as follows: Section 2 provides state-of-the-art literature related to some common topological multicast routing protocols that are commonly proposed for wireless adhoc networks. Section 3 provides details of ODMRP, as well as the basic and enhanced ToMuRo algorthm. Section 4 explains the scenario simulated and the results obtained. Finally, Section 5 summarizes our work and proposes future research. 


\section{State of the art of Multicast topological routing algorithms}

The Ad hoc Multicast Routing Protocol (AMRoute) presents a novel approach for robust IP Multicast in mobile ad hoc networks by exploiting usermulticast trees and dynamic logical cores. It creates a bidirectional, shared tree for data distribution using only group senders and receivers as tree nodes.

The main advantages of AMRoute are that it uses shared trees, requiring only one tree per group, which significantly improves its scalability. AMRoute is independent of the underlying unicast routing protocol. In addition, it floods a small signaling message instead of data. The major disadvantage of AMRoute is that it suffers from temporary loops and creates non-optimal trees when mobility is present [11]. In addition, upon tree creation, the core periodically unicasts TREE_CREATE messages to all mesh links. Other disadvantages of AMRoute are that it assumes the existence of an underlying unicast routing protocol that can be utilized for unicast IP communication between neighboring tree nodes and it periodically floods JOIN_REQ messages using an expanding ring search.

In ODMRP, group membership and multicast routes are established and updated by the source upon demand. Similar to on-demand unicast routing protocols, a request phase and a reply phase constitute the protocol. When a multicast source has packets to send, it floods a member advertising packet to neighbor nodes with data payload attached. This packet, called JOIN_DATA, is periodically broadcast to the entire network to refresh the membership information, thus taxing bandwidth resources. However, the advantage of ODMRP is that it performs well in terms of packet delivery ratio in highly dynamic environments [11], primarily because ODMRP provides route redundancy with a mesh topology that enables it to handle dynamic environments much better. Again, the main disadvantage in ODMRP is that group membership and multicast routes are established and updated by the source on demand, which can create congestion due to the significantly higher processing load the node must handle.

The main difference between AMRIS and other multicast routing protocols is that each participant in the multicast session must have a sessionspecific multicast session member id (msm-id). This $\mathrm{msm}$-id provides each node with an indication of its "logical height" in the multicast delivery tree.

The drawbacks of AMRIS are that each node must send a periodic beacon to signal their presence to neighboring nodes and that it is very sensitive to mobility and traffic load [11]. The primary reasons for its poor performance are the number of necessary retransmissions and the size of beacons, both of which create overhead and can cause increased congestion.

CAMP possesses good control traffic scalability to facilitate the increased size of a multicast group. Since JOIN_REQUESTS are only propagated until they reach a mesh member, CAMP does not incur exponential growth of multicast updates as the number of nodes and group members increase, which represents a significant advantage regarding bandwidth allocation and energy consumption. However, an important disadvantage of CAMP is that it employs a unicast routing protocol to handle network convergence and control traffic growth in the presence of mobility. Another major disadvantage of CAMP is that it assumes routing information from a unicast routing protocol is available and that the correct distance to the specified receiving node can be determined within a specified time. Finally, CAMP assumes that a wireless router contains a preexisting mapping service that provides group addresses which are identified by their specific names. 
The principal advantage of MAODV is that it employs the same RREQ/RREP messages as AODV. However, MAODV's main disadvantage is that it suffers from high End-to-End Delay (EED) [12] because packets must travel across longer discovery paths within the shared tree and because the higher network load caused by the larger number of control and data transmissions can cause increased congestion.

The advantages of ADMR are that it reduces the overhead of the routing protocol and improves its ability to react quickly to network topology changes. The main disadvantage of ADMR is its packet loss monitoring strategy, which can cause network congestion when it attempts to repair routes suffering from high packet loss [13].

Several publications compare the multicast routing algorithms mentioned previously [11], [12], [13], [14]. In [11], the authors compare AMRoute, ODMRP, AMRIS, CAMP, and Flooding. They report that AMRoute performs well in stationary conditions, but it suffers from loops and inefficient trees under even minimal mobility. AMRIS is effective with light traffic loads and no mobility, but its performance is significantly affected by heavier traffic loads and moderate mobility. CAMP shows better performance when compared to tree protocols, but increased mobility causes excessive control overhead, resulting in congestion and consequent performance degradation. ODMRP is very effective and efficient in most simulation scenarios. However, this protocol shows a tendency to rapidly increase overhead as the number of senders increases. In [12], the authors compare ADMR, ODMRP and MAODV. ADMR generates up to 14 times less control packet overhead than MAODV and up to 5 times less overhead than ODMRP. The high control overhead in MAODV is due to periodic flooding by the group leader and the significantly greater number of neighbor "Hello" packets. ODMRP's high overhead results from its periodic source flood and response cycles, with the response part of the cycle growing proportionally to the number of receivers. In [13], authors compare ODMRP and ADMR. ADMR induces higher overhead as node speed increases, because ADMR tries to repair routes as packet loss increases. Authors in [14], compare AMRIS and ODMRP. ODMRP delivers a higher percentage of packets correctly received when compared to AMRIS; around $20 \%$ for lower node speeds, and around $70 \%$ when node speed increases. This trend confirms that ODMRP is more robust than AMRIS.

This paper compares flooding and ODMRP with the basic and enhanced ToMuRO routing algorithm because literature reports that ODMRP possesses one of the most effective topological multicast routing algorithms and because Flooding is the simplest multicast mechanism.

\section{Topological multicast routing algorithms}

\section{A) On-Demand Multicast Routing Protocol (ODMRP)}

In ODMPR, group membership and multicast routes are established and updated by the source on demand. Similar to on-demand unicast routing protocols, ODMPR has both a request phase and a reply phase. When a multicast source sends packets, it uses a flooding strategy to transmit a member advertising packet to all the members of the group. This packet, called JOIN_DATA, which also carries the payload, is periodically broadcast to the entire network to refresh the membership information and update routes (Figure 1). When a node receives a non-duplicate JOIN_ DATA, it stores the upstream node ID into the routing table and rebroadcasts the packet. When the JOIN_ DATA packet reaches a multicast receiver, the receiver creates and broadcasts a JOIN_TABLE to its neighbors. 


\section{A Novel Topological Multicast Routing Algorithm (ToMuRo), R. Aquino-Santos et al., 44-55}

When a node receives a JOIN_TABLE, it verifies that the next node ID of one of the entries matches its own ID (Figure 2). If it does, the node realizes that it is located at an intermediate point between the source and receiver and recognizes that it must forward the packet. It then sets the FG_FLAG (Forwarding Group Flag) and broadcasts its own JOIN_TABLE based on matched entries. The JOIN_TABLE is thus propagated by each forwarding group member until it reaches the multicast source via the shortest path. This process constructs (or updates) the routes from sources to receivers and builds a mesh of nodes [6].

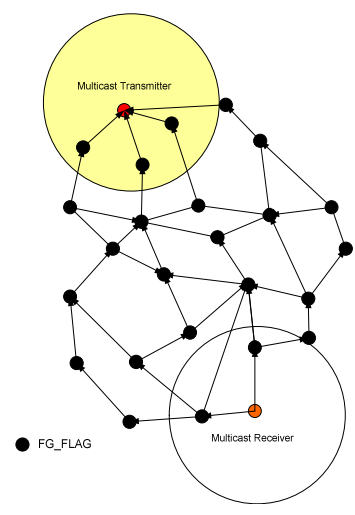

Figure 2. JOINT_TABLE Packet

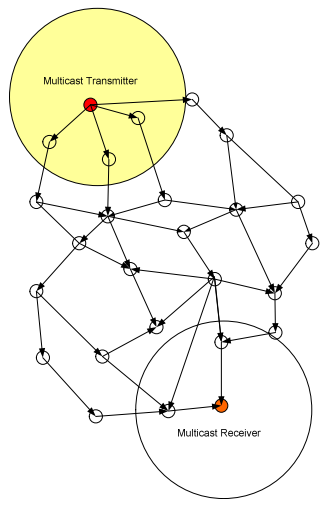

Figure 1. JOINT_DATA Packet

\section{B) Basic Topological Multicast Routing (ToMuRo)}

In the basic ToMuRo algorithm, group membership and multicast routes are established and updated by the receiver on demand, in which multicast request and reply phases constitute the protocol (Figure 3).

When a terminal wishes to receive a multicast packet, it floods a multicast request packet throughout the network. If a node within the transmission range of the multicast transmitter receives the multicast request packet, it replies back with a multicast reply packet.

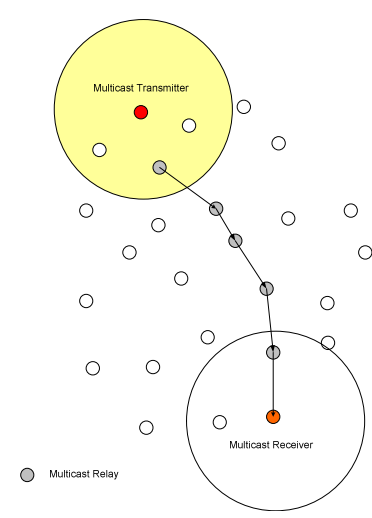

Figure 3. Multicast request and reply packets. 
Figure 4 shows the basic ToMuRo topological routing algorithm, which has four states: undecided, multicast relay, multicast receiver, and multicast transmitter. The multicast transmitter always sends packets in broadcast mode and multicast relay nodes retransmit the data packets.

The undecided nodes are employed to avoid retransmissions of unwanted multicast data packets. In their routing tables, undecided nodes record if they can reach the multicast transmitter and the last time they listened to it. When a multicast receiver wishes to receive a multicast data packet, it broadcasts a multicast request packet. If an undecided node receives the multicast request packet and it has a fresh route to the multicast transmitter, it replies a multicast reply packet. The multicast reply packet is sent back through the path formed with the multicast request packet.
C) Enhanced Topological Multicast Routing (ToMuRo)

In the enhanced version of ToMuRo, there are five states: undecided, multicast relay, multicast receiver, multicast transmitter, and undecided border node. Undecided nodes are employed to avoid retransmissions of unwanted data packets, similar to the basic ToMuRo. Also, the multicast transmitter always sends data packets in broadcast mode and the multicast relay nodes retransmit the data packets. The main difference between the basic ToMuRo and the enhanced ToMuRo is the undecided border nodes (Figure 5). The undecided border nodes are nodes that can listen to data packets and act as potential relay nodes, thus reducing the direction and time a packet requires to reach a node that is one hop from the multicast transmitter. Also, this mechanism may reduce possible collisions in dense networks because the packet has to traverse a shorter path.

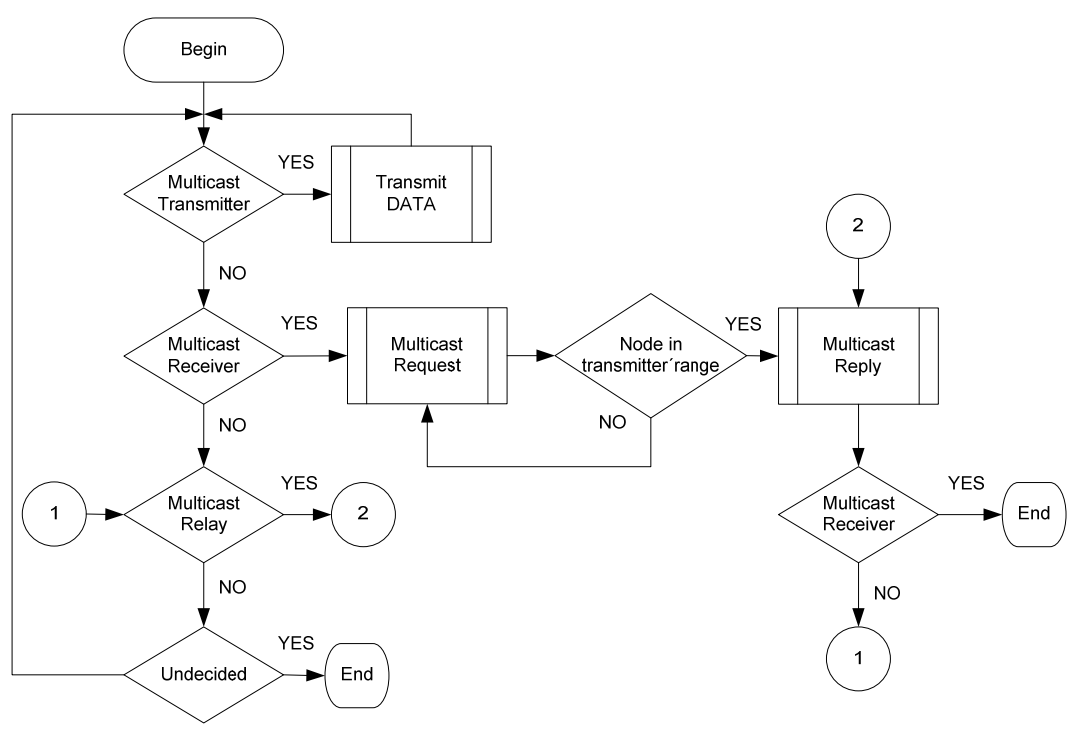

Figure 4. Flow diagram of the topological multicast routing algorithm. 


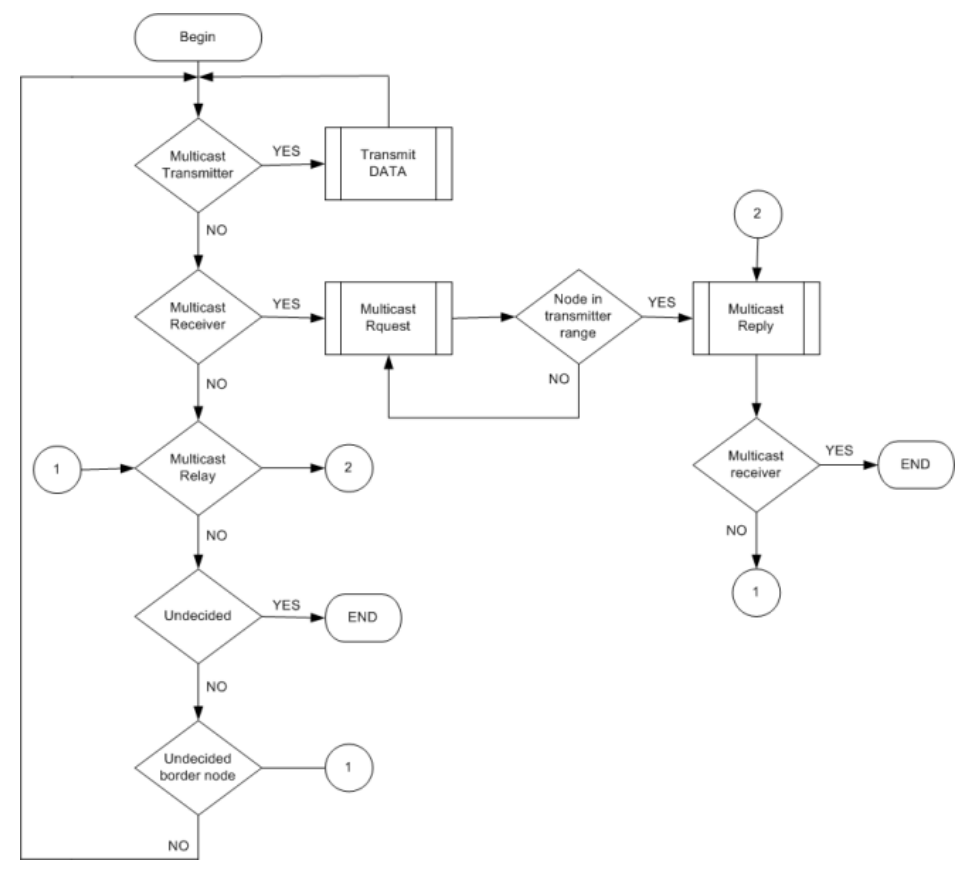

Figure 5. Flow diagram of the enhanced topological multicast routing algorithm.

Figure 5 illustrates the enhanced topological multicast routing algorithm. This flow diagram shows the undecided border node at the end, that is the difference between the basic and the extended multicast routing algorithm. The use of the undecided border node does not require a discovery process because it selects undecided nodes which can function as multicast relay nodes.

\section{Scenario simulated}

Flooding, ODMRP, basic, and enhanced ToMuRo were simulated using OPNET Modeler. OPNET Modeler is an important network simulator that is used to design and study communication networks, devices, protocols, and applications. Our simulation modeled a wireless network of 250 wireless nodes uniformly distributed within a $1200 \mathrm{~m} \times 1200 \mathrm{~m}$ area. Wireless node movements are based on the random-waypoint model. We used the IEEE 802.11b MAC protocol with an 11 Mbps channel capacity and a simulation time of
100 seconds. A pause time of 1 second was also applied. The scenario considers one multicast transmitter and one, two, and three multicast receivers under various mobility patterns and transmission ranges. In the simulation, node speeds of $0,5,10,15$, and 20 meters per second were chosen, and a constant bit rate (CBR) for data flow and a uniform payload size of 512 bytes. The simulation parameters are listed in Table I.

\begin{tabular}{|l|l|}
\hline Parameter & Value \\
\hline Simulation area & $1200 \mathrm{~m} \times 1200 \mathrm{~m}$ \\
\hline Total nodes & 250 \\
\hline Movement model & Random-waypoint model \\
\hline Channel capacity & $11 \mathrm{Mbps}$ \\
\hline Maximum speed & $0,5,10,15,20 \mathrm{~m} / \mathrm{sec}$ \\
\hline Pause time & 1 second \\
\hline MAC protocol & IEEE $802.11 \mathrm{~b}$ \\
\hline Packet flows & Constant bit rate (CBR) \\
\hline Packet payload & 512 bytes \\
\hline
\end{tabular}

Table 1. Simulation parameters. 


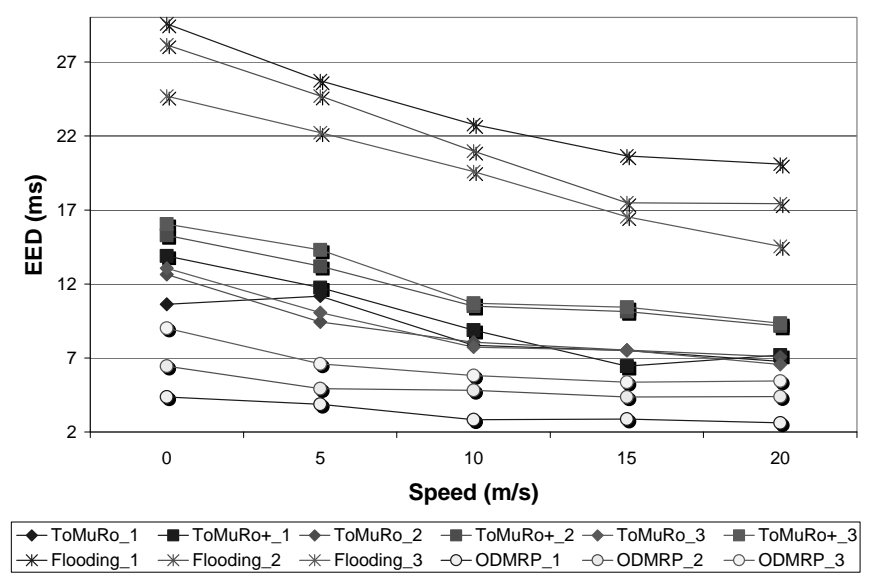

Figure 6. End-to-End Delay (EED).

Figure 6 represents the End-to-End Delay (EED); the horizontal line indicates the node speed in $\mathrm{m} / \mathrm{s}$ and the numbers in the labels under the graph (Flooding_1, Flooding_2, etc.) represent the number of receivers under simulated conditions. In general, flooding creates more End-to-End Delay. In this scenario, basic and enhanced ToMuRo show similar behavior because the multicast receivers were collocated in opposite positions. Basic and enhanced ToMuRo illustrate a little bit more EED than ODMRP.

Figure 7 shows the jitter for the multicast routing algorithms. Jitter is a critical variable for applications that are sensitive to delay because excessive Jitter can cause phase distortion during packet reception. Enhanced ToMuRo shows the best performance, and Flooding reports good results in terms of jitter. The performance of Basic ToMuRo improves as node speed increases. On the other hand, the performance DMRP improves when the number of multicast receivers increases.

Figure 8 represents the percentage of packets received. In contrast to the previous figures, ODMRP performs poorly with only one multicast receiver. Flooding improves its behavior as the number of receiver decreases, but it still does not perform as well as enhanced ToMuRo. The performance of basic ToMuRo is satisfactory when the number of multicast receiver increases.

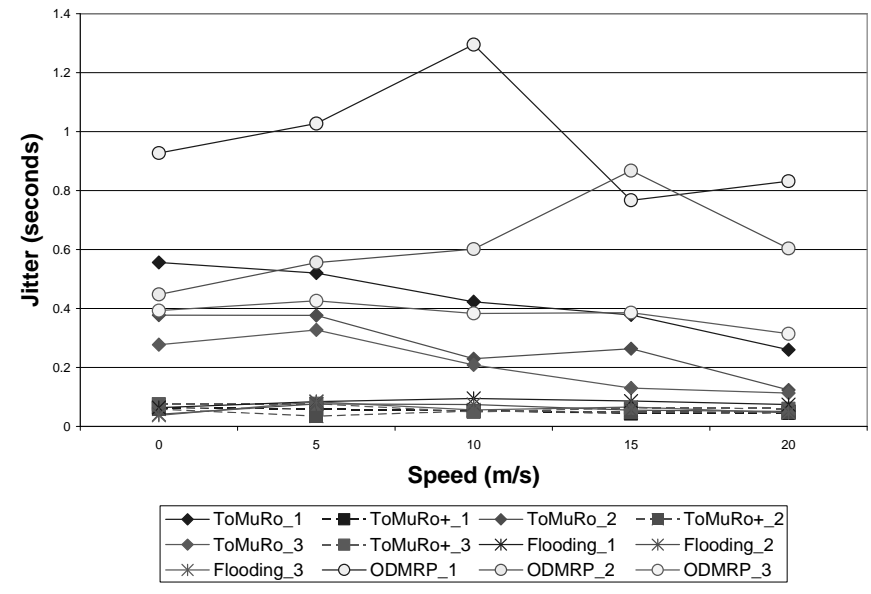

Figure 7. Jitter for the multicast routing algorithms. 


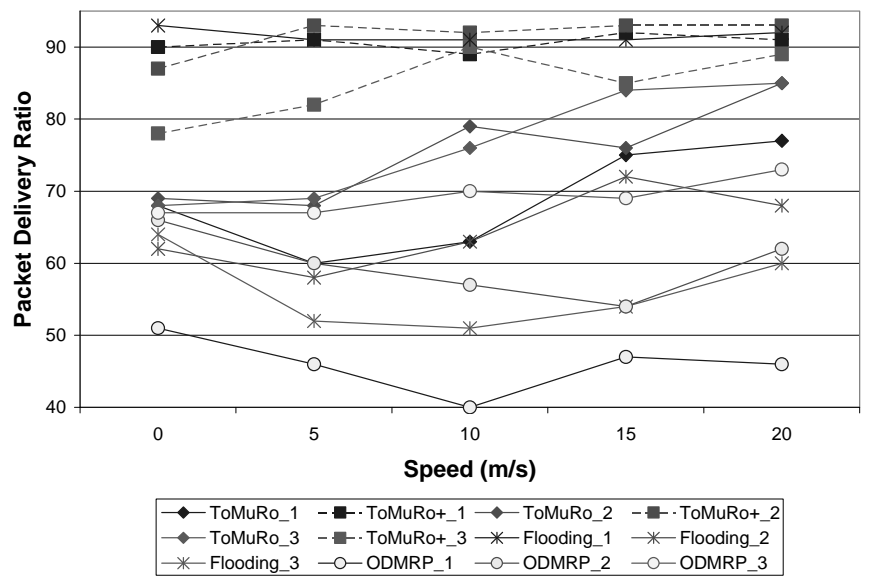

Figure 8. Packet delivery ratio for the multicast routing algorithms.

Figure 9, represents the data transmitted. This metric shows the efficiency of the algorithm in retransmitting data packets throughout the network. Flooding shows the worst behavior of the four algorithms because of its data packet retransmission mechanism. Basic and enhanced ToMuRo have similar behavior for one receiver, but for two and three receivers, ODMRP performs slightly worse than ToMuRo.

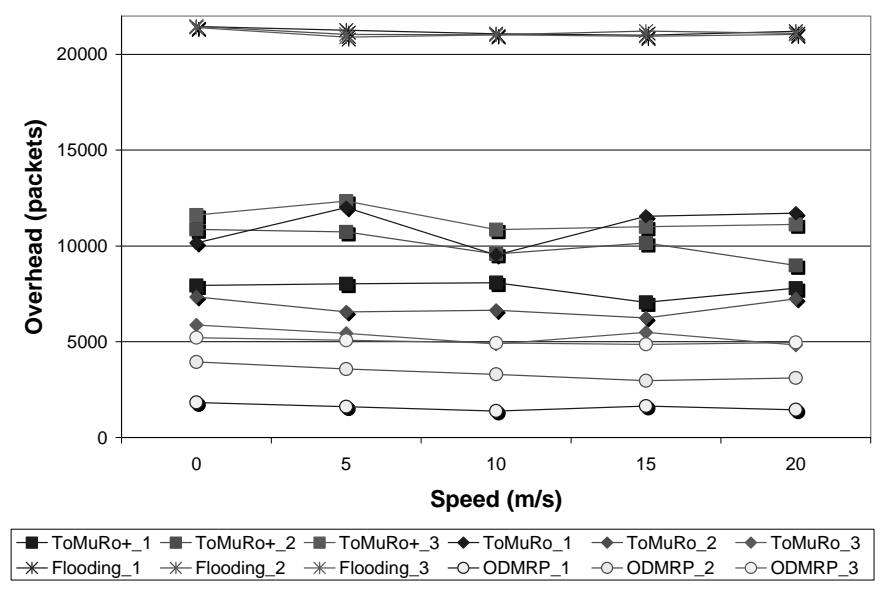

Figure 9. Overhead for the multicast routing algorithms. 


\section{Conclusions}

This paper has presented three multicast routing protocols. Simulation results show that the enhanced ToMuRo algorithm performs better than the ODMRP algorithm in terms of jitter and packet delivery ratio. However, enhanced ToMuRo performs slightly worse in terms of EED and Overhead. The performance of enhanced ToMuRo, when compared with ODMRP, is better in terms of two very important QoS parameters for applications (jitter and packet delivery ratio). However, in terms of EED, both algorithms perform well. It conclusion enhanced ToMuRo may be more appropriate for multimedia or other applications that require streaming than ODMRP and Basic ToMuRo.

Our future work will simulate geographical multicast routing algorithms under higher node mobility.

\section{References}

[1] Deering, S. P., Cheriton, D. R. Multicast Routing in Datagram Inter-networks and Extended LANs. ACM Transactions on Computer Systems, vol 8, no. 2, pp. 85110, 1990.

[2] Moy, J. Multicast Routing Extensions for OSPF. Communications of the ACM, vol. 37 , no. 8 , pp. 61-66, 1994.

[3] Ballardie, T., Francis, P., Crowcroft, J. Core Based Trees (CBT) - An Architecture for Scalable Inter-Domain Multicast Routing. In Proceedings of ACM SIGCOMM'93, pp. 85-95, 1993.

[4] Deering, D., Estrin, D. L., Farinacci, D., Jacobson, V., Liu, C. G. Wei, L. The PIM Architecture for Wide-Area Multicast Routing. IEEE/ACM Transactions on Networing, vol. 4, no.2, pp. 153-162, 1999.
[5] Xie, J., Talpade, R., Mcauley, A., Liu, M. AMRoute: Ad Hoc Multicast Routing Protocol.Mobile Networks and Applications, vol. 7, no. 6, pp. 429-439, 2002.

[6] Sang, H. B., Sung-ju, L., William, S., Gerla, M. The Design, Implementation, and Performance Evaluation of the On-Demand Multicast Routing Protocol in Multihop Wireless Networks. IEEE Network, pp. 70-77, 2000.

[7] Wu, C. W., Tay, Y. C. AMRIS: A Multicast Protocol for Ad hoc Wireless Networks. Proceedings of IEEE MILCOM'99. pp. 25-29, 1999.

[8] Garcia-Luna-Aceves, J.J., Madruga, E. L. The CoreAssisted Mesh Protocol. IEEE Journal on Selected Areas in Communications, vol. 17, no. 8,pp. 151-165, 1999.

[9] Royer, E. M., Perkins, Ch. E. Multicast Operation of the Ad hoc On-Demand Distance Vector Routing Protocol. Mobile Computing and Networking, pp. 207218, 1999.

[10]Jetcheva, J. G., Jonson, D. B. Adaptive DemandDriven Multicast Routing in Multi-Hop Wireless Ad Hoc Networks. Proceedings of the second symposium on Mobile Ad Hoc Networking and Computing (MobiHoc 2001), pp. 33-44, 2001.

[11]Lee, S., Su, W., Gerla, M., Bagrodia, R. A Performance Comparison Study of Ad Hoc Wireless Multicast Protocols. Proceedings of the IEEE INFOCOM, pp. 565-574, 2000.

[12] Jetcheva, J. G., Jonson, D. B. A Performance Comparison of On-Demand Multicast Routing Protocols for Ad Hoc Networks. CMU-CS-04-176, 2004.

[13] Pandey, M., Zappala, D. A scenario-Based Performance Evaluation of Multicast Routing for Ad Hoc Networks. Proceeding of the Sixth IEEE International Symposium on a World of Wireless Mobile and Multimedia Networks (WoWMoM'05), pp. 31-41, 2005.

[14] Malaguti, M., Taddia, C., Mazzini, G., Zorzi, M. Analysis of Performance of Multicast Routing Protocols over 802.11b, IEEE Vehicular Technology Conference, vol, 5, pp. 3165-3169, 2004. 


\section{Authors' Biography}

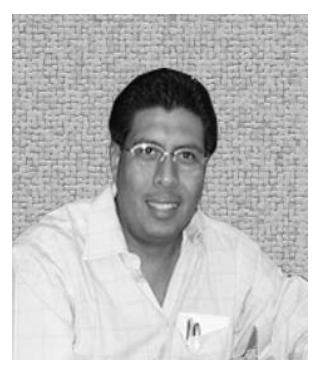

\section{Raúl AQUINO-SANTOS}

He graduated from the Universidad de Colima with a B.E. degree in electrical engineering, received his M.S. degree in telecommunications from the Centre for Scientific Research and Higher Education in Ensenada, Mexico in 1990. He holds a Ph.D. degree from the Department of Electrical and Electronic Engineering of the University of Sheffield, England. Since 2005, he has been with the College of Telematics, at the Universidad de Colima, where he is currently a research professor in telecommunications networks. His current research interests include wireless and sensor networks.

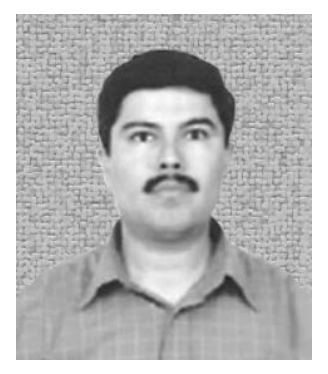

\section{Armando Luis VILLASEÑOR-GONZALEZ}

He is currently a faculty member of the Electronics and Telecommunications Department at CICESE Research Center. He received his Ph.D. degree in electrical engineering from the University of Ottawa, Canada in 2002. His current research interests include wireless communication networks, QoS protocol architectures, performance analysis and evaluation of Internet technologies and computer networks. He is currently a member of the IEEE.

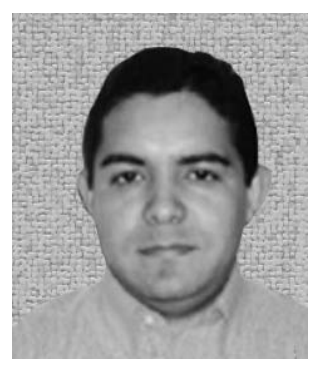

\section{Victor RANGEL-LICEA}

He received the B.Eng (Hons) degree in computer engineering in the Engineering Faculty from the Universidad Nacional Autónoma de México (UNAM) in 1996, the M.Sc degree in telematics from the University of Sheffield, U.K. in 1998, and the Ph.D. degree in performance analysis and traffic scheduling in cable networks in 2002, from the University of Sheffield. Since 2002, he has been with the School of Engineering, UNAM, where he is currently a research professor in telecommunications networks. His research focuses on fixed, mesh and mobile broadband wireless access networks, QoS over IP, traffic shaping and scheduling. 

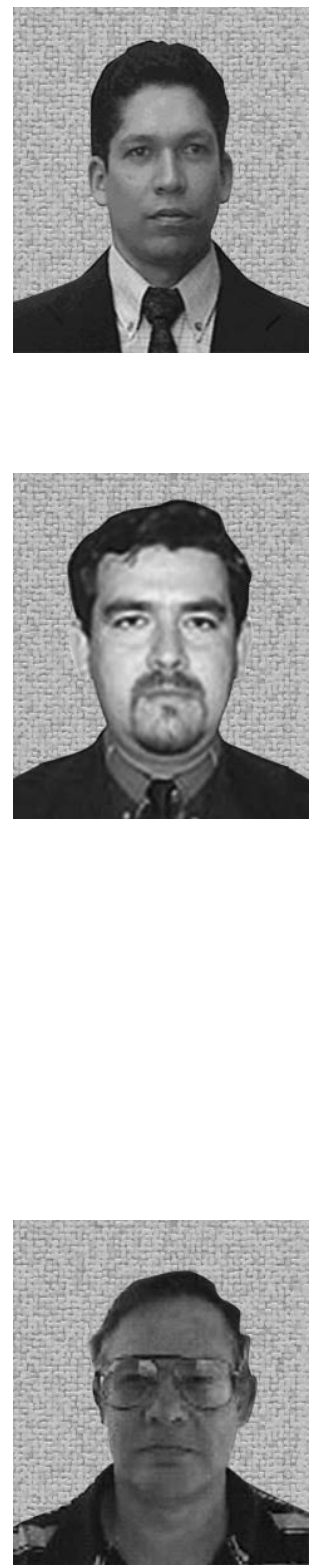

\section{Apolinar GONZALEZ-POTES}

He holds a Ph.D. degree from Universidad Politécnica de Valencia, Spain. Since 2004, he has been with the Faculty of Mechanical Electrical Engineering of the Universidad de Colima, where he is currently a research-professor in informatics. His current research interest includes mobile computing and embedded software.

\section{Miguel A. GARCIA-RUIZ}

He graduated in computer systems engineering and obtained his M.Sc degree in computer science from the Universidad de Colima, Mexico. He received his Ph.D. in computer science and Artificial Intelligence from the School of Cognitive and Computing Sciences, University of Sussex, England. He took a virtual reality course at Salford University, England and a graphics techniques internship at the Universidad Politécnica de Madrid, Spain. Miguel has been a visiting professor at the University of Ontario, Institute of Technology, Canada. He has been teaching computer science courses, and doing research mainly on virtual reality and multimodal interfaces at the Universidad de Colima. Miguel has published various scientific papers in major journals and a book and has directed a video documentary about an introduction to virtual reality.

\section{Arthur Edwards}

He received his masters degree in education from the University of Houston in 1985. He has been a research professor at the Universidad de Colima since 1985 , where he has served in various capacities. He has been with the School of Telematics since 1998. His primary areas of research are Computer Assisted Language Learning (CALL), distance learning, collaborative learning, multimodal leaning and mobile learning. The primary focus of his research is presently in the area of mobile collaborative learning. 